\title{
Bayesian using Importance Sampling Technique of Weibull Regression with Type II Censored Data
}

\author{
Mohammed Ahmed Al Omari
}

\begin{abstract}
Keeping in view the Bayesian approach, the study aims to develop methods through the utilization of Jeffreys prior and modified Jeffreys prior to the covariate obtained by using the Importance sampling technique. For maximum likelihood estimator, covariate parameters, and the shape parameter of Weibull regression distribution with the censored data of Type II will be estimated by the study. It is shown that the obtained estimators in closed forms are not available, but through the usage of appropriate numerical methods, they can be solved. The mean square error is the criterion of comparison. With the use of simulation, performances of these three estimates are assessed, bearing in mind different censored percentages, and various sizes of the sample.
\end{abstract}

Index Terms - Bayesian method, Survival and Hazard functions, Importance Sampling Technique, Modified Jeffreys prior, Type II censoring.

\section{INTRODUCTION}

Generally, survival models with the Bayesian approach are a bit difficult to fit, but via the usage of techniques, for instance, MCMC, survival models like fitting complex can be made straightforward. Also, it is easier to implement the software's availability. For that, we have constructed in this study the Markov Chain Monte Carlo (MCMC) method. In Bayesian, importance sampling is implemented to numerically calculate posterior distributions that frequently comprise of integrals so that the deductions can be made. Also, with its usage, credible Bayesian intervals could be constructed. One reason why importance sampling is pursued enthusiastically is due to the struggle in drawing samples straight from the wanted marginal posterior distributions. Therefore, the approach of importance sampling needs to be used for evaluation of the posterior densities, particularly when direct sampling from the wanted posterior position does not seem possible, see Chen \& Shao [1] and Gelfand and Smith [2]. Importance sampling was used by Johns [3] for obtaining bootstrap confidence intervals and applying it for obtaining credible Bayesian intervals, for details sees Hinkley [4]. Goffinet \& Wallach [5] established a method of effective importance sampling to estimate the test statistic quantiles. The Bayesian model of the inverse Weibull distribution was considered by Howlader \& Kundu [6] for censored data of Type-II, where the usage of the technique of importance sampling occurs for estimating the parameters of shape and scale, the credible intervals, and survival function.

Prior distribution got categories, one of them is Jeffreys's prior information for data with no available prior information; it is very beneficial. Alomari et al [7] consider the Bayesian with Jeffreys prior and extension of Jeffrey's prior estimation, and for more detail, see Sinha [8] and Sun [9]. Chen et al. [10] established an algorithm of importance sampling under Jeffreys prior. When Jeffrey's prior applied to panel models whose effects are fixed, they produce posterior inference that comes with an incidental parameter problem, as shown in Hahn [11]. Singh et al. [12] estimated the generalized maximum likelihood estimator, the Bayesian estimators, and the maximum likelihood estimator via non-dependent priors of noninformative types were measured for the three unknown parameters. Shrinkage for the scale parameter of an exponential distribution under censored data of type-II where the non-informative prior was attained, estimated by Prakash and Singh [13]. Calabria and Pulcini [14], Grodecki [15], and Lu and Zhu [16] estimated the Bayesian model with Jeffreys prior for estimating the parameters.

The objective of this study is to incorporate the covariate to Jeffrey's prior and develop the Jeffrey's prior in power function and called modified Jeffrey's prior. After that, estimate the covariate parameters, survival function, shape parameter, and hazard function of the Weibull regression distribution with censoring data of Type- II by using the Importance Sampling Technique.

Published on June 23, 2021

Mohammed Ahmed Al Omari, Department of Mathematics, Faculty of Arts and Sciences in Qilwah, AlBaha University, Baha, Saudi Arabia.

(corresponding e-mail: aurangak @yahoo.com) 


\section{Methodology}

\section{A. Maximum Likelihood Estimation of Weibull Censored Data}

The maximum likelihood estimation of Weibull distribution based on type II censored data can be introduced by let be the set of random lifetime from Weibull distribution with scale parameter and shape parameter. The probability density function of Weibull distribution is:

$$
f(\mathrm{t} ; \lambda, \alpha)=\frac{\alpha}{\lambda} t^{\alpha-1} \exp \left(-\frac{t^{\alpha}}{\lambda}\right)
$$

and the cumulative distribution function is:

$$
F(\mathrm{t} ; \lambda, \alpha)=1-\exp \left(-\frac{t^{\alpha}}{\lambda}\right)
$$

where $\lambda$ is the scale parameter and $\alpha$ is the shape parameter, then the likelihood function is:

$$
L(\lambda, \alpha \mid \mathbf{t})=\frac{n !}{(n-r) !}\left[\prod_{i=1}^{r} \frac{\alpha}{\lambda} t_{i}^{\alpha-1} \exp \left(-\frac{t_{i}^{\alpha}}{\lambda}\right)\right]\left[\exp \left(-\frac{t_{r}^{\alpha}}{\lambda}\right)\right]^{n-r}
$$

The covariates were incorporated to the scale parameter as follows [17]:

$$
\begin{gathered}
\lambda=\exp \left(\beta^{\prime} x_{i}\right) \text { the } x_{i}=\left(1, x_{i 1}, x_{i 2}, \ldots, x_{i n}\right) \text { is the vector of covariates, } \\
\text { and } \beta^{\prime}=\left(\beta_{0}, \beta_{1}, \beta_{2}, \ldots, \beta_{n}\right) \text { is the coefficient vector. }
\end{gathered}
$$

Therefore, the likelihood function can be written as:

$L\left(t ; \beta^{\prime}, \alpha\right)=\frac{n !}{(n-r) !}\left[\prod_{i=1}^{r} \alpha t_{i}^{\alpha-1} \exp \left(-\beta^{\prime} x_{i}-t_{i}^{\alpha} \exp \left(-\beta^{\prime} x\right)\right)\right]\left[\exp \left(-t_{r}^{\alpha} \exp \left(-\beta^{\prime} x\right)\right)\right]^{n-r}$

The $\log$ - likelihood function can be written as:

$\ln L(\lambda, \alpha \mid x)=\log \frac{n !}{(n-r) !}+r \log (\alpha)+(\alpha-1) \sum_{i=1}^{r} \log \left(\mathrm{t}_{i}\right)-\sum_{i=1}^{r} \beta^{\prime} x_{i}+t_{i}^{\alpha} \exp \left(-\beta^{\prime} x_{i}\right)+(n-r)\left(-t_{r}^{\alpha} \exp \left(-\beta^{\prime} x_{i}\right)\right)$

The resulting equations are given as:

$$
\begin{aligned}
& \frac{\partial \ln L(\beta, \alpha)}{\partial \beta_{j}}=-\sum_{i=1}^{r} x_{i}+x_{i j} t^{\alpha} \exp \left(-\beta^{\prime} x_{i}\right)+(n-r)\left(-x_{r} t^{\alpha} \exp \left(-\beta^{\prime} x_{i}\right)\right) \\
& \frac{\partial L(\beta, \alpha)}{\partial \alpha}=\frac{r}{\alpha}+r \log (t)+\sum_{i=1}^{r} \log \left(t_{i}\right)-\sum_{i=1}^{r} t_{i}^{\alpha} \log \left(t_{i}\right) \exp \left(-\beta^{\prime} x_{i}\right)+(n-r)\left(-t_{r}^{\alpha} \log \left(t_{r}\right) \exp \left(-\beta^{\prime} x_{i}\right)\right)
\end{aligned}
$$

The estimates of the survival and hazard functions are:

$$
\begin{aligned}
& \hat{S}_{M}(t)=\exp \left(-t^{\hat{\alpha}_{M}} \exp \left(-\hat{\beta}_{M}^{\prime} x_{i}\right)\right) \\
& \hat{h}_{M}(t)=\hat{\alpha}_{M} \exp \left(-\hat{\beta}_{M}^{\prime} x_{i}\right) t^{\hat{\alpha}_{M}-1}(5)
\end{aligned}
$$

where $\hat{\beta}_{M}^{\prime}$ are the parameters of covariate estimation by MLE and $\hat{\alpha}_{M}$ is shape parameter estimation by MLE. The equations ( 2 to 5) cannot be solved it analytically, therefore, we are using Newton Raphson iterative approach to solve the equations.

\section{B. Bayesian using Jeffreys Prior of Covariate Estimation}

In our prior distribution we need to find the Fisher information matrix as follows: 


$$
I\left(\beta^{\prime}, \alpha\right)=\left(\begin{array}{ccccc}
n x_{1} & \cdots & \sum x_{n} & n\left(\beta_{0}+\Gamma^{\prime}(2)\right) / \alpha \\
\sum x_{1} & \sum x_{1}^{2} & \cdots & \sum x_{1} x_{n} & \sum_{i=1}^{n} x_{i 1}\left(\beta_{1} x_{i 1}+\Gamma^{\prime}(2)\right) / \alpha \\
\cdots & \cdots & \cdots & \cdots & \cdots \\
\sum x_{n} & \sum x_{1} x_{n} & \cdots & \sum x_{n}^{2} & \cdots \\
n\left(\beta_{0}+\Gamma^{\prime}(2)\right) / \alpha & \sum_{i=1}^{n} x_{i 1}\left(\beta_{1} x_{i 1}+\Gamma^{\prime}(2)\right) / \alpha & \cdots & \cdots & \sum_{i=1}^{n} \frac{1}{\alpha^{2}}\left[\delta_{i}+\left(\beta^{\prime} x_{i}\right)^{2}+\Gamma^{\prime \prime}(2)+2 \beta^{\prime} x_{i} \Gamma^{\prime}(2)\right]
\end{array}\right)
$$

where the Jeffreys prior with covariate is:

$$
g_{1}\left(\beta^{\prime}, \alpha\right)=k \sqrt{\operatorname{det}\left(I\left(\beta^{\prime}, \alpha\right)\right)}
$$

The posterior density function is,

$$
\prod_{B J}\left(\beta^{\prime}, \alpha \mid t\right)=\frac{\sqrt{\operatorname{det}\left(I\left(\beta^{\prime}, p\right)\right)}}{J_{1}}\left[\prod_{i=1}^{r} \alpha t_{i}^{\alpha-1} \exp \left(-\beta^{\prime} x_{i}-t_{i}^{\alpha} \exp \left(-\beta^{\prime} x\right)\right)\right][\mathrm{u}]^{n-r}(7)
$$

where

$$
\begin{aligned}
& J_{1}=\int_{0}^{\infty} \ldots \int_{0}^{\infty} \sqrt{\operatorname{det}\left(I\left(\beta^{\prime}, p\right)\right)}\left[\prod_{i=1}^{r} \alpha t_{i}^{\alpha-1} \exp \left(-\beta^{\prime} x_{i}-t_{i}^{\alpha} \exp \left(-\beta^{\prime} x\right)\right)\right]\left[\exp \left(-t_{i}^{\alpha} \exp \left(-\beta^{\prime} x\right)\right)\right]^{n-r} d \beta^{\prime} d \alpha \\
& \mathrm{u}=\exp \left(-t_{i}^{\alpha} \exp \left(-\beta^{\prime} x\right)\right)
\end{aligned}
$$

Bayesian estimator under squared error loss function of parameters of covariate is:

$$
\hat{\beta}_{B J}=\frac{1}{J_{1}} \int_{0}^{\infty} \cdots \int_{0}^{\infty} \beta_{j} \sqrt{\operatorname{det}\left(I\left(\beta^{\prime}, \alpha\right)\right)}\left[\prod_{i=1}^{r} \alpha t_{i}^{\alpha-1} \exp \left(-\beta^{\prime} x_{i}-t_{i}^{\alpha} \exp \left(-\beta^{\prime} x\right)\right)\right][u]^{n-r} d \beta^{\prime} d \alpha
$$

Bayesian estimator under squared error loss function of shape parameter is:

$$
\hat{\alpha}_{B J}=\frac{1}{J_{1}} \int_{0}^{\infty} \ldots \int_{0}^{\infty} \alpha \sqrt{\operatorname{det}\left(I\left(\beta^{\prime}, \alpha\right)\right)}\left[\prod_{i=1}^{r} \alpha t_{i}^{\alpha-1} \exp \left(-\beta^{\prime} x_{i}-t_{i}^{\alpha} \exp \left(-\beta^{\prime} x\right)\right)\right][u]^{n-r} d \beta^{\prime} d \alpha
$$

The survival function of the Weibull regression based on type II censored data as shown below:

$$
\hat{S}(t)=\frac{1}{J_{1}} \int_{0}^{\infty} \cdots \int_{0}^{\infty} \exp \left(-t^{\alpha} \exp \left(-\beta^{\prime} x_{i}\right)\right) \sqrt{\operatorname{det}\left(I\left(\beta^{\prime}, \alpha\right)\right)}\left[\prod_{i=1}^{r} \alpha t_{i}^{\alpha-1} \exp \left(-\beta^{\prime} x_{i}-t_{i}^{\alpha} \exp \left(-\beta^{\prime} x\right)\right)\right][u]^{n-r} d \beta^{\prime} d \alpha
$$

The hazard function of the Weibull regression based on type II censored data as shown below:

$$
\hat{h}(t)=\frac{1}{J_{1}} \int_{0}^{\infty} \ldots \int_{0}^{\infty} \alpha t^{\alpha-1} \exp \left(-\beta^{\prime} x_{i}\right) \sqrt{\operatorname{det}\left(I\left(\beta^{\prime}, \alpha\right)\right)}\left[\prod_{i=1}^{r} \alpha t_{i}^{\alpha-1} \exp \left(-\beta^{\prime} x_{i}-t_{i}^{\alpha} \exp \left(-\beta^{\prime} x\right)\right)\right][u]^{n-r} d \beta^{\prime} d \alpha
$$

The equations (8 to 11) we cannot solve it analytically. However, we can use Importance Sampling Technique for the estimation.

\section{Modified Jeffreys of Covariate Estimation}

The modified Jeffreys prior is the Fisher information matrix with covariate in power function as given below:

$$
g_{2}\left(\beta^{\prime}, \alpha\right)=k\left(\operatorname{det}\left[I\left(\beta^{\prime}, \alpha\right)\right]\right) \log ^{\log \left(\sum_{j=0}^{n} \beta_{j}\right)}
$$


The posterior probability density function of modified Jeffreys prior with covariate for parameters of covariate $\beta^{\prime}$ and shape parameter $p$ given the data $\left(t_{1}, \ldots, t_{n}\right)$ is taken by divide the joint probability density function over marginal density function as follows

$$
\prod_{B M}\left(\beta^{\prime}, \alpha \mid t\right)=\frac{\left(\operatorname{det}\left[I\left(\beta^{\prime}, \alpha\right)\right]\right)^{\log \left(\sum_{j=0}^{n} \beta_{j}\right)}}{J_{2}}\left[\prod_{i=1}^{r} \alpha t_{i}^{\alpha-1} \exp \left(-\beta^{\prime} x_{i}-t_{i}^{\alpha} \exp \left(-\beta^{\prime} x\right)\right)\right][u]^{n-r}
$$

where

$$
J_{2}=\int_{0}^{\infty} \cdots \int_{0}^{\infty}\left(\operatorname{det}\left[I\left(\beta^{\prime}, \alpha\right)\right]\right)^{\log \left(\sum_{j=0}^{n} \beta_{j}\right)}\left[\prod_{i=1}^{r} \alpha t_{i}^{\alpha-1} \exp \left(-\beta^{\prime} x_{i}-t_{i}^{\alpha} \exp \left(-\beta^{\prime} x\right)\right)\right][u]^{n-r} d \beta^{\prime} d \alpha
$$

Bayesian using modified Jeffreys prior estimator under squared error loss function is:

$$
\hat{\beta}_{B M}=\frac{1}{J_{2}} \int_{0}^{\infty} \ldots \int_{0}^{\infty} \beta_{j}\left(\operatorname{det}\left[I\left(\beta^{\prime}, \alpha\right)\right]\right)^{\log \left(\sum_{j=0}^{n} \beta_{j}\right)}\left[\prod_{i=1}^{r} \alpha t_{i}^{\alpha-1} \exp \left(-\beta^{\prime} x_{i}-t_{i}^{\alpha} \exp \left(-\beta^{\prime} x\right)\right)\right][u]^{n-r} d \beta^{\prime} d \alpha
$$

Bayesian using modified Jeffreys prior estimator under squared error loss function to estimate shape parameter with covariate is:

$\hat{\alpha}_{B M}=\frac{1}{J_{2}} \int_{0}^{\infty} \ldots \int_{0}^{\infty} \alpha\left(\operatorname{det}\left[I\left(\beta^{\prime}, \alpha\right)\right]\right)^{\log \left(\sum_{j=0}^{n} \beta_{j}\right)}\left[\prod_{i=1}^{r} \alpha t_{i}^{\alpha-1} \exp \left(-\beta^{\prime} x_{i}-t_{i}^{\alpha} \exp \left(-\beta^{\prime} x\right)\right)\right][u]^{n-r} d \beta^{\prime} d \alpha$

The survival function of the Weibull regression with modified Jeffreys prior is:

$\hat{S}_{M B}(t)=\frac{1}{J_{2}} \int_{0}^{\infty} \ldots \int_{0}^{\infty} \exp \left(-t^{\alpha} \exp \left(-\beta^{\prime} x_{i}\right)\right)\left(\operatorname{det}\left[I\left(\beta^{\prime}, \alpha\right)\right]\right) \log \left(\sum_{j=0}^{n} \beta_{j}\right)\left[\prod_{i=1}^{r} \alpha t_{i}^{\alpha-1} \exp \left(-\beta^{\prime} x_{i}-t_{i}^{\alpha} \exp \left(-\beta^{\prime} x\right)\right)\right][u]^{n-r} d \beta^{\prime} d \alpha$

The hazard function of the Weibull regression with modified Jeffreys prior is:

$$
\hat{h}_{B M}(t)=\frac{1}{J_{2}} \int_{0}^{\infty} \ldots \int_{0}^{\infty} \alpha t^{\alpha-1} \exp \left(-\beta^{\prime} x_{i}\right)\left(\operatorname{det}\left[I\left(\beta^{\prime}, \alpha\right)\right]\right)^{\log \left(\sum_{j=0}^{n} \beta_{j}\right)}\left[\prod_{i=1}^{r} \alpha t_{i}^{\alpha-1} \exp \left(-\beta^{\prime} x_{i}-t_{i}^{\alpha} \exp \left(-\beta^{\prime} x\right)\right)\right][u]^{n-r} d \beta^{\prime} d \alpha
$$

We cannot solve the equations (13) to (16) analytically in Bayesian for modified Jeffreys prior. However, we can use Importance Sampling Technique for the estimation.

\section{Importance Sampling for Jeffreys Prior with Covariate}

The full conditional of the posterior density function of and for Bayesian using Jeffreys prior with covariate given the data can be written as:

$$
\prod_{1}\left(\beta^{\prime}, \alpha ; t\right) \propto \sqrt{\operatorname{det}\left(I\left(\beta^{\prime}, \alpha\right)\right)}\left[\alpha^{n}\left(\prod_{i=1}^{n} t_{i}^{\alpha-1}\right) \exp \left(-\sum_{i=1}^{n} \beta^{\prime} x_{i}\right) \exp \left(-\sum_{i=1}^{n} t_{i}^{\alpha} \exp \left(-\beta^{\prime} x_{i}\right)\right)\right][u]^{n-r}
$$

Equation (17) of the posterior of the parameters of covariate and shape parameter used the importance sampling technique as follows:

$$
\prod_{1}\left(\beta^{\prime}, \alpha \mid t\right) \propto \pi\left(\beta^{\prime} \mid \alpha, t\right) \pi(\alpha \mid t) h_{1}\left(\beta^{\prime}, \alpha \mid t\right)
$$


See, Kundu and Howlader [6].

The conditional posterior of the parameter of covariate given the shape parameter and the data is as follows:

$$
\pi\left(\beta^{\prime} \mid \alpha, t\right) \propto \sum_{i=1}^{n} x_{i j} \exp \left(-\beta^{\prime} \sum_{i=1}^{n} x_{i j}\right)
$$

The conditional posterior of parameters of covariate, given the shape parameter and the data, follows the exponential distribution with parameter $\sum_{i=1}^{n} x_{i j}$, and the conditional posterior of the shape parameter given the data is given below:

$$
\pi(\alpha \mid t) \propto \operatorname{gamma},\left(n+1, \sum_{i=1}^{n} \log t_{i}\right)
$$

and

$$
h_{1}\left(\beta^{\prime}, \alpha \mid t\right) \propto \sqrt{\operatorname{det}\left(I\left(\beta^{\prime}, \alpha\right)\right)} \exp \left(-\sum_{i=1}^{n} t_{i}^{\alpha} \exp \left(-\beta^{\prime} x_{i}\right)\right)\left(\exp \left(-t_{i}^{\alpha} \exp \left(-\beta^{\prime} x\right)\right)\right)^{n-r}
$$

The Bayesian estimation of parameters of covariate and shape parameter under the squared error loss function is:

$$
\Upsilon_{1}\left(\beta^{\prime}, \alpha\right)=\frac{\int_{0}^{\infty} \cdots \int_{0}^{\infty} \omega_{1}\left(\beta^{\prime}, \alpha\right) \pi\left(\beta^{\prime} \mid \alpha, t\right) \pi(\alpha \mid t) h_{1}\left(\beta^{\prime}, \alpha \mid t\right) d \beta^{\prime} d \alpha}{\int_{0}^{\infty} \cdots \int_{0}^{\infty} \pi\left(\beta^{\prime} \mid \alpha, t\right) \pi(\alpha \mid t) h_{1}\left(\beta^{\prime}, \alpha \mid t\right) d \beta^{\prime} d \alpha}
$$

The equation above cannot be solved analytically. For that we propose to estimate it via importance sampling technique as shown in Algorithm 1.

Algorithm 1:

1) Generate the shape parameter $\alpha_{c}$ from gamma density function with $\left(n+1, \sum_{i=1}^{n} \log t_{i}\right)$.

2) Generate parameters of covariate $\beta^{\prime}$ for Bayesian using Jeffreys prior from Exponential distribution with parameter $\sum_{i=1}^{n} x_{i j}$

3) Repeat steps 1 and 2, $\mathrm{N}$ times to get $\beta_{i}^{\prime}, \alpha_{c i}$

4) The Bayesian estimation of parameters of covariate and shape, say under the squared error loss function is:

$$
\hat{\Upsilon}_{1}\left(\beta^{\prime}, p\right)=\hat{\mu}=\frac{\sum_{i=1}^{n} \mu_{i} h_{1}\left(\beta_{i}^{\prime}, \alpha_{c i} \mid t\right)}{\sum_{i=1}^{n} h_{1}\left(\beta_{i}^{\prime}, \alpha_{c i} \mid t\right)}
$$

where $\mu$ is the general case of the parameters of covariate and shape.

5) The posterior variance of Bayesian using Jeffreys prior is $\hat{V}\left(\hat{\mu}_{M J}\right)=\frac{\sum_{i=1}^{N}\left(\mu_{i}-\hat{\mu}_{M J}\right)^{2} h_{2}\left(\beta_{i}^{\prime}, \alpha_{u i} \mid t\right)}{\sum_{i=1}^{N} h_{2}\left(\beta_{i}^{\prime}, \alpha_{u i} \mid t\right)}$

6) The survival and hazard functions with covariate for Bayesian using modified Jeffreys prior with covariate are as follows:

$$
\hat{S}_{\beta 1}^{*}(t)=\frac{\sum_{i=1}^{N} \exp \left(t_{j}^{\alpha_{c i}} \exp \left(-\beta^{\prime} x_{i}\right)\right) h_{1}\left(\beta_{i}^{\prime}, \alpha_{c i} \mid t\right)}{\sum_{i=1}^{N} h_{1}\left(\beta_{i}^{\prime}, \alpha_{c i} \mid t\right)} .
$$




$$
\hat{h}_{\beta 1}^{*}(t)=\frac{\sum_{i=1}^{N} \alpha_{c i} t_{j}^{\alpha_{c i}-1} \exp \left(-\beta^{\prime} x_{i}\right) h_{1}\left(\beta_{i}^{\prime}, \alpha_{c i} \mid t\right)}{\sum_{i=1}^{N} h_{1}\left(\beta_{i}^{\prime}, \alpha_{c i} \mid t\right)}
$$

\section{E. Importance Sampling for Modified Jeffreys Prior with Covariate}

The full conditional of the posterior density function of parameters of covariate and shape using modified Jeffreys prior with covariate given the data is:

$$
\prod_{2}\left(\beta^{\prime}, \alpha \mid t\right) \propto\left(\operatorname{det}\left[I\left(\beta^{\prime}, \alpha\right)\right]\right)^{\log \left(\sum_{j=0}^{n} \beta_{j}\right)}\left[\prod_{i=1}^{r} \alpha t_{i}^{\alpha-1} \exp \left(-\beta^{\prime} x_{i}-t_{i}^{\alpha} \exp \left(-\beta^{\prime} x\right)\right)\right][u]^{n-r}
$$

The importance sampling technique as follows:

$$
\prod_{2}\left(\beta^{\prime}, \alpha \mid t\right) \propto \pi\left(\beta^{\prime} \mid \alpha, t\right) \pi(\alpha \mid t) h_{2}\left(\beta^{\prime}, \alpha \mid t\right) .
$$

Bayesian using modified Jeffreys prior estimation of and say under the squared error loss function is:

$$
\Upsilon_{2}\left(\beta^{\prime}, \alpha\right)=\frac{\int_{0}^{\infty} \cdots \int_{0}^{\infty} \omega_{1}\left(\beta^{\prime}, p\right) \pi\left(\beta^{\prime} \mid \alpha, t\right) \pi(\alpha \mid t) h_{2}\left(\beta^{\prime}, \alpha \mid t\right) d \beta^{\prime} d \alpha}{\int_{0}^{\infty} \cdots \int_{0}^{\infty} \pi\left(\beta^{\prime} \mid \alpha, t\right) \pi(\alpha \mid t) h_{2}\left(\beta^{\prime}, \alpha \mid t\right) d \beta^{\prime} d \alpha} .
$$

The conditional posterior of the $\beta^{\prime}$ and $\alpha$ given the data $h_{2}\left(\beta^{\prime}, \alpha \mid t\right)$ is given as below:

$$
h_{2}\left(\beta^{\prime}, \alpha \mid t\right) \propto\left(\operatorname{det}\left[I\left(\beta^{\prime}, \alpha\right)\right)^{\log \left(\sum_{j=0}^{n} \beta_{j}\right)} \exp \left(-\sum_{i=1}^{n} t_{i}^{\alpha} \exp \left(-\beta^{\prime} x_{i}\right)\right)[u]^{n-r}\right.
$$

and the $\pi\left(\beta^{\prime} \mid \alpha, t\right)$ is in the equation (19) and the $\pi(\alpha \mid t)$ is given in the equation (20).

Here again we propose to estimate $\beta^{\prime}$ and $\alpha$ via importance sampling technique as shown in Algorithm 2. Algorithm 2:

1) Generate the shape parameter $\alpha_{u}$ from gamma density function with $\left(n+1, \sum_{i=1}^{n} \log t_{i}\right)$

2) Generate parameters of covariate $\beta^{\prime}$ of Bayesian using modified Jeffreys prior from Exponential distribution with parameter. $\sum_{i=1}^{n} x_{i j}$

3) Repeat steps 1 and $2 N$ times.

4) The Bayesian estimation of $\mu_{M J}$ under the SELF is given as $\hat{\Upsilon}_{2}\left(\beta^{\prime}, \alpha\right)=\hat{\mu}_{M J}=\frac{\sum_{i=1}^{N} \mu_{i} h_{2}\left(\beta_{i}^{\prime}, \alpha_{u i} \mid t\right)}{\sum_{i=1}^{N} h_{2}\left(\beta_{i}^{\prime}, \alpha_{u i} \mid t\right)}$

5) Obtain the posterior variance of Bayesian using modified Jeffreys prior as follows:

6)

$$
\hat{V}\left(\hat{\mu}_{M J}\right)=\frac{\sum_{i=1}^{N}\left(\mu_{i}-\hat{\mu}_{M J}\right)^{2} h_{2}\left(\beta_{i}^{\prime}, \alpha_{u i} \mid t\right)}{\sum_{i=1}^{N} h_{2}\left(\beta_{i}^{\prime}, \alpha_{u i} \mid t\right)}
$$

7) The survival and hazard functions with covariate for Bayesian using modified Jeffreys prior with covariate are as follows:

$$
\hat{S}_{\beta 2}^{*}(t)=\frac{\sum_{i=1}^{N} \exp \left(t_{j}^{\alpha_{\text {ui }}} \exp \left(-\beta^{\prime} x_{i}\right)\right) h_{2}\left(\beta_{i}^{\prime}, \alpha_{u i} \mid t\right)}{\sum_{i=1}^{N} h_{2}\left(\beta_{i}^{\prime}, \alpha_{u i} \mid t\right)} .
$$




$$
\hat{h}_{\beta 2}^{*}(t)=\frac{\sum_{i=1}^{N} \alpha_{u i} t_{j}^{\alpha_{c i}-1} \exp \left(-\beta^{\prime} x_{i}\right) h_{1}\left(\beta_{i}^{\prime}, \alpha_{u i} \mid t\right)}{\sum_{i=1}^{N} h_{1}\left(\beta_{i}^{\prime}, \alpha_{u i} \mid t\right)} .
$$

\section{SimUlation STUDY}

To provide a simulation of maximum likelihood estimation with covariate and Bayesian by using Jeffreys's prior and modified Jeffreys's prior with covariate. By using the Importance sampling Technique, we generate a lifetime from Weibull regression distribution with different sample sizes and diverse censoring percentage.

The maximum likelihood estimation is by using equations (2) and (3), respectively, for estimating shape parameter and covariate parameters. The hazard functions and survival are estimated from equations (4) and (5), respectively.

The parameter of scale is equal to $\exp \left(B_{0}+B_{1} x_{1}+B_{2} x_{2}\right)$ with the initial values $\left(\beta_{0}=2, \beta_{1}=1, \beta_{2}=-1\right)$ and the value of shape parameter is 3 .

The Bayesian using Jeffreys prior with covariate for estimation of the shape and covariate parameters of the Weibull regression model, follow algorithms one and estimate the parameters of shape and covariate of Weibull regression distribution via Bayesian with modified Jeffreys prior as shown in Algorithm 2.

Estimate the Weibull regression model's hazard functions and survival by Bayesian using Jeffreys prior with covariate from Algorithm 1 and the Bayesian using modified Jeffreys prior for estimation of the hazard functions and survival of Weibull regression distribution can be obtained from Algorithm 2.

The number of repetitions was 10,000 times, and for each repetition, mean square error (MSE) of covariate and shape parameter, the hazard function and survival function of Weibull regression was estimated for each method. In Tables I-VI, results are displayed for the different percentages of censoring and sample size.

\section{RESUlTS AND DISCUSSION}

As shown in Tables I to IV the parameters of the covariates and the parameter of the shape of Weibull regression with censored data of Type II estimated by Bayesian using Jeffreys prior (BJ), Maximum likelihood, and Bayesian using modified Jeffreys prior (BM) via importance sampling technique, keeping in mind the mean square error (MSE).

For the parameters of the covariates and the shape parameter, the Bayesian model has a smaller mean square error (MSE) than Maximum likelihood. The modified Jeffreys prior of covariate give better results than Jeffreys with the covariates for all cases.

The maximum likelihood estimator of the parameters of covariates for the mean square error is calculated and obtained for $\beta_{0}$ as 1.671 for a sample size of 30 and $r=15$. Having repeated the sample for 10,000 times, the mean square error of parameters of covariates was determined. The modified Jeffreys priors and Bayesian estimator using Jeffreys aimed at estimation of the covariate's parameters regarding the mean square error and was also obtained for $\beta_{1}$ as 0.0330 and 0.0327 respectively for a sample of size 30 with $r=15$.

In Table $\mathrm{V}$, when we compare the estimated survival function of Weibull regression based on censored data of Type II regarding the mean square error (MSE). The result shows that the Bayesian using modified Jeffreys prior and Jeffreys prior is better than the Maximum Likelihood Estimator (MLE) except when the sample size was 20 and $r=15$ the maximum likelihood estimation was better than the Bayesian using Jeffreys prior. However, the Bayesian using Jeffreys prior is less good than the Bayesian using modified Jeffreys prior.

In Table VI, the Weibull regression hazard function based on censored data of Type II was compared regarding mean square error (MSE). Result tells that, Bayesian using modified Jeffreys prior gives smaller values compared to the other estimations except when the sample size was 50 with $r=25$, as compared to others, the maximum likelihood estimation was better. 
TABLE I: ESTIMATOR THE PARAMETER OF $\beta_{0}$ WITH (MSE)

\begin{tabular}{ccccc}
\hline$n$ & $r$ & MLE & BJ & BM \\
\hline \multirow{3}{*}{30} & 15 & $1.671(0.0372)$ & $1.676(0.0369)$ & $2.313(0.0364)$ \\
& 20 & $1.680(0.0370)$ & $1.682(0.0366)$ & $2.305(0.0361)$ \\
& 25 & $1.686(0.0369)$ & $1.684(0.0365)$ & $2.301(0.0360)$ \\
\hline \multirow{3}{*}{40} & 20 & $1.673(0.0371)$ & $1.679(0.0368)$ & $2.308(0.0363)$ \\
& 30 & $1.681(0.0367)$ & $1.685(0.0365)$ & $2.302(0.0361)$ \\
& 35 & $1.684(0.0366)$ & $1.689(0.0363)$ & $2.291(0.0360)$ \\
\hline \multirow{2}{*}{50} & 25 & $1.678(0.0370)$ & $1.682(0.0367)$ & $2.305(0.0362)$ \\
& 35 & $1.686(0.0364)$ & $1.687(0.0363)$ & $2.294(0.0359)$ \\
& 45 & $1.689(0.0363)$ & $1.692(0.0358)$ & $2.289(0.0354)$ \\
\hline
\end{tabular}

TABLE II: ESTIMATOR THE PARAMETER OF $\beta_{1}$ WITH (MSE)

\begin{tabular}{ccccc}
\hline$N$ & $r$ & MLE & BJ & BM \\
\hline \multirow{3}{*}{30} & 15 & $0.832(0.0331)$ & $0.834(0.0330)$ & $1.161(0.0327)$ \\
& 20 & $0.835(0.0329)$ & $0.837(0.0328)$ & $1.159(0.0325)$ \\
& 25 & $0.838(0.0327)$ & $0.841(0.0326)$ & $1.156(0.0321)$ \\
\hline \multirow{3}{*}{40} & 20 & $0.842(0.0324)$ & $0.846(0.0321)$ & $1.151(0.0319)$ \\
& 30 & $0.845(0.0322)$ & $0.848(0.0319)$ & $1.148(0.0318)$ \\
& 35 & $0.847(0.0320)$ & $0.850(0.0318)$ & $1.145(0.0317)$ \\
\hline \multirow{2}{*}{50} & 25 & $0.851(0.0318)$ & $0.857(0.0313)$ & $1.141(0.0311)$ \\
& 35 & $0.852(0.0317)$ & $0.860(0.0312)$ & $1.143(0.0310)$ \\
& 45 & $0.855(0.0315)$ & $0.864(0.0310)$ & $1.142(0.0309)$ \\
\hline
\end{tabular}

TABLE III: ESTIMATOR THE PARAMETER OF $\beta_{2}$ WITH (MSE)

\begin{tabular}{ccccc}
\hline$N$ & $r$ & MLE & BJ & BM \\
\hline \multirow{3}{*}{30} & 15 & $-1.159(0.0287)$ & $-1.158(0.0286)$ & $-0.865(0.0284)$ \\
& 20 & $-1.152(0.0284)$ & $-1.151(0.0282)$ & $-0.871(0.0279)$ \\
& 25 & $-1.149(0.0283)$ & $-1.147(0.0281)$ & $-0.874(0.0278)$ \\
\hline \multirow{3}{*}{40} & 20 & $-1.141(0.0274)$ & $-1.140(0.0273)$ & $-0.891(0.0269)$ \\
& 30 & $-1.137(0.0272)$ & $-1.135(0.0271)$ & $-0.895(0.0268)$ \\
& 35 & $-1.132(0.0270)$ & $-1.131(0.0269)$ & $-0.900(0.0266)$ \\
\hline \multirow{2}{*}{50} & 25 & $-1.121(0.0262)$ & $-1.118(0.0258)$ & $-0.921(0.0255)$ \\
& 35 & $-1.115(0.0259)$ & $-1.112(0.0257)$ & $-0.925(0.0254)$ \\
& 45 & $-1.111(0.0258)$ & $-1.108(0.0256)$ & $-0.931(0.0252)$ \\
\hline
\end{tabular}

TABLE IV: ESTIMATOR THE PARAMETER OF $\alpha$ WITH (MSE)

\begin{tabular}{ccccc}
\hline$n$ & $r$ & MLE & BJ & BM \\
\hline \multirow{3}{*}{30} & 15 & $2.571(0.0231)$ & $2.576(0.0230)$ & $3.413(0.0227)$ \\
& 20 & $2.580(0.0229)$ & $2.582(0.0227)$ & $3.405(0.0224)$ \\
& 25 & $2.586(0.0228)$ & $2.590(0.0226)$ & $3.401(0.0223)$ \\
\hline \multirow{3}{*}{40} & 20 & $2.591(0.0226)$ & $2.593(0.0224)$ & $3.396(0.0219)$ \\
& 30 & $2.596(0.0224)$ & $2.597(0.0223)$ & $3.392(0.0217)$ \\
& 35 & $2.601(0.0223)$ & $2.602(0.0221)$ & $3.390(0.0216)$ \\
\hline \multirow{3}{*}{50} & 25 & $2.609(0.0219)$ & $2.611(0.0218)$ & $3.384(0.0213)$ \\
& 35 & $2.613(0.0217)$ & $2.615(0.0215)$ & $3.381(0.0212)$ \\
& 45 & $2.614(0.0216)$ & $2.617(0.0214)$ & $3.378(0.0211)$ \\
\hline
\end{tabular}

TABLE V: MEAN SQUARE ERROR OF ESTIMATES THE SURVIVAL FUNCTION With T=0.5

\begin{tabular}{ccccc}
\hline$n$ & $r$ & MLE & BJ & BM \\
\hline \multirow{3}{*}{30} & 15 & 0.0151 & 0.0152 & 0.0149 \\
& 20 & 0.0149 & 0.0148 & 0.0147 \\
& 25 & 0.0148 & 0.0146 & 0.0145 \\
\hline \multirow{3}{*}{40} & 20 & 0.0150 & 0.0146 & 0.0143 \\
& 30 & 0.0148 & 0.0145 & 0.0142 \\
& 35 & 0.0146 & 0.0144 & 0.0141 \\
\hline \multirow{2}{*}{50} & 25 & 0.0144 & 0.0142 & 0.0139 \\
& 35 & 0.0142 & 0.0140 & 0.0137 \\
& 45 & 0.0140 & 0.0139 & 0.0136 \\
\hline
\end{tabular}

TABLE VI: MEAN SQUARE ERROR OF ESTIMATES THE HAZARD FUNCTION WITH T=0.5

\begin{tabular}{ccccc}
\hline$n$ & $r$ & MLE & BJ & BM \\
\hline \multirow{3}{*}{30} & 15 & 0.7531 & 0.7530 & 0.7527 \\
& 20 & 0.7529 & 0.7528 & 0.7525 \\
& 25 & 0.7528 & 0.7527 & 0.7523 \\
\hline \multirow{3}{*}{40} & 20 & 0.7525 & 0.7526 & 0.7521 \\
& 30 & 0.7523 & 0.7523 & 0.7520 \\
& 35 & 0.7521 & 0.7520 & 0.7518 \\
\hline \multirow{2}{*}{50} & 25 & 0.7515 & 0.7516 & 0.7517 \\
& 35 & 0.7514 & 0.7513 & 0.7512 \\
& 45 & 0.7509 & 0.7510 & 0.7509 \\
\hline
\end{tabular}




\section{CONCLUSION}

According to the Weibull regression model based on censored data of Type II, Jeffreys prior and modified Jeffreys prior information was used in Bayesian by using the Importance sampling technique are better estimators compared to Maximum Likelihood Estimator (MLE). However, modified Jeffreys prior used in the Bayesian via Importance sampling technique are better than Jeffreys prior. Our results indicated that, with different percentages of censoring, there is a change for the estimator parameters of covariate, shape parameter, survival, and hazard functions. All the methods produced smaller of the mean square error as the sample size increases, as indicated in the simulation study.

\section{REFERENCES}

[1] Chen, M. H., \& Shao, Q. M. (1999). Monte Carlo estimation of Bayesian credible and HPD intervals. Journal of Computational and Graphical Statistics, , 69-92.

[2] Gelfand, A. E., \& Smith, A. F. M. (1990). Sampling-based approaches to calculating marginal densities. Journal of the American Statistical Association, 85(410), 398-409.

[3] Johns, M. V. (1988). Importance sampling for bootstrap confidence intervals. Journal of the American Statistical Association, 83(403), 709-714

[4] Hinkley, D. V. (1988). Bootstrap methods. Journal of the Royal Statistical Society.Series B (Methodological), $321-337$.

[5] Goffinet, B., \& Wallach, D. (1996). Optimised importance sampling quantile estimation. Biometrika, 83(4), 791-800.

[6] Kundu, D., \& Howlader, H. (2010) Bayesian inference and prediction of the inverse Weibull distribution for type-II censored data. Computational Statistics \& Data Analysis, 54 (6), 1547_1558.

[7] Al Omari, M. A. \& Ibrahim, N. A. \& Arasan, J. and Adam, M. B., (2012) Bayesian Survival and Hazard Estimate for Weibull Censored Time Distribution. Journal of Applied Sciences, 12: 1313-1317.

[8] Sinha, S. K. (1986). Bayes estimation of the reliability function and hazard rate of a Weibull failure time distribution. Trabajos De Estadística, 1(2), 47-56.

[9] Sun, D. (1997). A note on noninformative priors for Weibull distributions. Journal of Statistical Planning and Inference, 61(2), 319-338.

[10] Chen, M. H., Ibrahim, J. G., \& Kim, S. (2008). Properties and implementation of Jeffreys's prior in Binomial regression models. Journal of the American Statistical Association, 103(484), 1659-1664.

[11] Hahn, J. (2004). Does Jeffreys's prior alleviate the incidental parameter problem? Economics Letters, 82(1), $135-138$.

[12] Singh, U., Gupta, P. K., \& Upadhyay, S. K. (2005). Estimation of parameters for Exponentiated-Weibull family under type-II censoring scheme. Computational Statistics \& Data Analysis, 48(3), 509-523.

[13] Prakash, G., \& Singh, D. C. (2008). Shrinkage estimation in Exponential type-II censored data under LINEX loss. Journal of the Korean Statistical Society, 37(1), 53-61.

[14] Calabria, R., \& Pulcini, G. (1994). An engineering approach to Bayes estimation for the Weibull distribution. Microelectronics and Reliability, 34(5), 789-802.

[15] Grodecki, J. (2001). Generalized maximum-likelihood estimation of variance-covariance components with non-informative prior. Journal of Geodesy, 75(2), 157-163.

[16] Zhu, M., \& Lu, A. Y. (2004). The counter-intuitive non-informative prior for the Bernoulli family. Journal of Statistics Education, 12(2), 1-10.

[17] Kleinbaum D. G. \& Klein M. (2005). Survival Analysis: A Self-Learning Text. Springer.

[18] Al Omari, M. A. (2016). Bayesian Study Using MCMC of Gompertz Distribution Based on Interval Censored Data with Three Loss Functions. Journal of applied sciences, 16: 88-97.

[19] Soliman, A. A., Abd-Ellah, A. H., Abou-Elheggag, N. A., \& Ahmed, E. A. (2011). Modified Weibull model: A Bayes study using MCMC approach based on progressive censoring data. Reliability Engineering \& System Safety, Volume 100, April 2012, Pages 48-57. 